PROCEEDINGS OF THE

AMERICAN MATHEMATICAL SOCIETY

Volume 139, Number 4, April 2011, Pages 1263-1271

S 0002-9939(2010)10569-5

Article electronically published on August 31, 2010

\title{
A UNIVERSAL CHARACTERIZATION OF THE CHERN CHARACTER MAPS
}

\author{
GONÇALO TABUADA
}

(Communicated by Brooke Shipley)

\begin{abstract}
The Chern character maps are one of the most important working tools in mathematics. Although they admit numerous different constructions, they are not yet fully understood at the conceptual level. In this paper we eliminate this gap by characterizing the Chern character maps, from the Grothendieck group to the (negative) cyclic homology groups, in terms of simple universal properties.
\end{abstract}

\section{INTRODUCTION}

In his foundational work, Chern 2] introduced character maps from $K$-theory to de Rham cohomology in order to study complex vector bundles on smooth manifolds. Chern's construction made use of connection forms, exterior derivatives, etc.

Forty years later, Connes 3 extended Chern's work to the non-commutative setting. He invented a new theory that plays the role of de Rham cohomology and constructed Chern character maps with values in it. Given a commutative base ring $k$ and a unital (but not necessarily commutative) $k$-algebra $A$, the (functorial) Chern character maps

$$
c h^{-}: K_{0}(A) \longrightarrow H C_{0}^{-}(A), \quad c h_{n}: K_{0}(A) \longrightarrow H C_{2 n}(A), n \geq 0
$$

go from the Grothendieck group to the (negative) cyclic homology groups; see 6 , $\S 8]$. Connes's construction made use of idempotents, a generalized trace map, etc.

Later, in the nineties, McCarthy [7] and Keller 5 extended 1 the Chern character maps (1.1) from $k$-algebras to dg categories; see $\$ 2$ for the notion of a dg category. Given a commutative and unital base ring $k$, we have the natural transformations

$$
c h^{-}: K_{0} \Rightarrow H C_{0}^{-} \quad c h_{n}: K_{0} \Rightarrow H C_{2 n}, n \geq 0
$$

between functors defined on the category of small $\mathrm{dg}$ categories and with values in abelian groups; see [7, §4.4], [5]. The construction of the Chern character maps (1.2) made use of simplicial $S^{1}$-fixed points, a generalized $S_{\bullet}$-construction, etc.

Received by the editors March 1, 2010 and, in revised form, April 22, 2010.

2010 Mathematics Subject Classification. Primary 19L10, 18D20, 19 D55.

Key words and phrases. Chern character maps, dg categories, (negative) cyclic homology.

The author was partially supported by the Estimulo à Investigação Award 2008 - Calouste Gulbenkian Foundation and by the FCT-Portugal grant PTDC/MAT/098317/2008.11.

${ }^{1} \mathrm{McCarthy}$ worked in the context of exact $k$-categories. Keller then improved McCarthy's definition of cyclic homology, making it available for dg categories.

(C)2010 American Mathematical Society Reverts to public domain 28 years from publication 
At this point one wonders if it is possible to isolate the fundamental properties of the Chern character maps while discarding specific constructions without losing any information. The natural question is the following:

How does one conceptually understand/characterize the Chern character maps (1.2)?

In this note we give an absolute and a relative solution to this question. In \$3, we provide a description of the canonical isomorphisms

$$
\psi^{-}: H C_{0}^{-}(k) \stackrel{\sim}{\longrightarrow} k, \quad \psi_{n}: H C_{2 n}(k) \stackrel{\sim}{\longrightarrow} k, n \geq 0 .
$$

Theorem 1.3 (Absolute characterization). The canonical maps

$$
\begin{aligned}
\operatorname{Nat}\left(K_{0}, H C_{0}^{-}\right) \stackrel{\sim}{\longrightarrow} k, & \eta \mapsto \psi^{-}(\eta(\underline{k})([k])), \\
\operatorname{Nat}\left(K_{0}, H C_{2 n}\right) \stackrel{\sim}{\longrightarrow} k, & \eta \mapsto \psi_{n}(\eta(\underline{k})([k])), n \geq 0
\end{aligned}
$$

are isomorphisms of abelian groups. Here, $\underline{k}$ stands for the dg category with a single object and with $k$ as the $(d g) k$-algebra of endomorphisms, $[k]$ stands for the class of $k$ (as a module over itself) in the Grothendieck group $K_{0}(\underline{k})=K_{0}(k)$, and Nat stands for the abelian group of natural transformations (with group structure given by objectwise addition).

Under the canonical isomorphisms (1.4) and (1.5), the Chern character maps $\mathrm{ch}^{-}$and $\mathrm{ch}_{n}$ (1.2) are characterized as the natural transformations corresponding to the unit 1 of the base ring $k$.

Recall from [6, §5.1.8] the sequence of natural transformations:

$$
H C_{0}^{-} \stackrel{I}{\Rightarrow} H C_{0}^{\mathrm{per}} \Rightarrow{\underset{\lim }{n}}_{n} H C_{2 n} \Rightarrow \cdots \stackrel{S}{\Rightarrow} H C_{2 n} \stackrel{S}{\Rightarrow} H C_{2 n-2} \Rightarrow \cdots \stackrel{S}{\Rightarrow} H C_{0}
$$

Theorem 1.7 (Relative characterization). Fix a Chern character map ch $_{m}$ (1.2).

(i) Then, the Chern character map $c h_{n}, n>m$, is the unique natural transformation such that $S^{(n-m)} \circ \mathrm{ch}_{n}=c h_{m}$.

(ii) Similarly, the Chern character map $\mathrm{ch}^{-}$is the unique natural transformation such that the composition

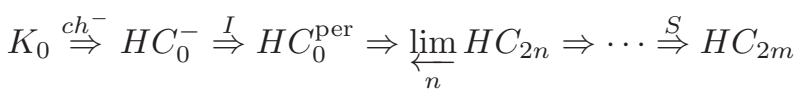

equals the Chern character map $\mathrm{ch}_{m}$.

To the best of the author's knowledge, Theorems 1.3 and 1.7 offer the first conceptual characterization of the Chern character maps. Theorem 1.3 characterizes the Chern character maps among all natural transformations, and Theorem 1.7 asserts that, up to compatibility with the sequence (1.6), there is only one Chern character map. It is expected that the conceptual understanding here presented will play a catalytic role in the several branches of mathematics where the Chern character maps are used. This will be the subject of future research.

Convention 1.9. In the sequel $k$ denotes a commutative base ring with unit 1. 


\section{AdDitive INVARIANTS OF DG CATEGORIES}

In this section we review and develop some of the ingredients of the theory of additive invariants of dg categories [9, 10, which will be used in the proofs of Theorems 1.3 and 1.7 .

A differential graded $(=d g)$ category (over the base ring $k$ ) is a category enriched over complexes of $k$-modules (Hom-sets are complexes) in such a way that the composition fulfills the graded Leibniz rule

$$
d(f \circ g)=(d f) \circ g+(-1)^{\operatorname{deg}(f)} f \circ(d g) .
$$

In particular, a dg category with a single object is simply a dg $k$-algebra. For a survey article on dg categories we invite the reader to consult Keller's ICM address 4. We denote by dgcat the category of small dg categories.

As in the case of a (dg) $k$-algebra, given a dg category $\mathcal{A}$ we can consider its derived category $\mathcal{D}(\mathcal{A})$ of right $\mathcal{A}$-modules; see [4, §3.1]. $\mathrm{A} \operatorname{dg}$ functor $\mathcal{A} \rightarrow \mathcal{B}$ is called a Morita equivalence if the restriction of scalar functors $\mathcal{D}(\mathcal{B}) \stackrel{\sim}{\rightarrow} \mathcal{D}(\mathcal{A})$ is an equivalence of (triangulated) categories; see [4, §3.8].

Let $\mathcal{A}$ be a dg category. Consider the dg category $T(\mathcal{A})$ whose objects are the pairs $(i, x)$, where $i$ is an element of the set $\{1,2\}$ and $x$ is an object of $\mathcal{A}$. The complex of morphisms in $T(\mathcal{A})$, from $(i, x)$ to $\left(i^{\prime}, x^{\prime}\right)$, is given by $\mathcal{A}\left(x, x^{\prime}\right)$ if $i^{\prime} \geq i$ and is 0 otherwise. Composition is induced by the composition operation in $\mathcal{A}$; see [9, §4] for details. Note that we have two natural inclusion dg functors

$$
i_{1}: \mathcal{A} \longrightarrow T(\mathcal{A}), \quad i_{2}: \mathcal{A} \longrightarrow T(\mathcal{A}) \text {. }
$$

Definition 2.1. Let $E:$ dgcat $\rightarrow \mathrm{D}$ be a functor with values in an additive category. We say that $E$ is an additive invariant if it satisfies the following two conditions:

(i) it sends the Morita equivalences to isomorphisms;

(ii) given any $\operatorname{dg}$ category $\mathcal{A}$, the inclusion $\operatorname{dg}$ functors induce an isomorphism 2

$$
\left[E\left(i_{1}\right) E\left(i_{2}\right)\right]: E(\mathcal{A}) \oplus E(\mathcal{A}) \stackrel{\sim}{\longrightarrow} E(T(\mathcal{A})) .
$$

A morphism of additive invariants is a natural transformation of functors. We denote by $\operatorname{Fun}_{\mathrm{A}}($ dgcat, D) the category of additive invariants with values in D.

Example 2.2. Examples of additive invariants with values in (the additive category of) abelian groups include the Grothendieck group functor $\left(K_{0}\right)$, the cyclic homology group functors $\left(H C_{n}, n \geq 0\right)$, the zero periodic homology group functor $\left(H C_{0}^{\text {per }}\right)$, the zero negative homology group functor $\left(H C_{0}^{-}\right)$, etc.; see [9, $\left.\S \S 6.1-6.2\right]$.

As in the case of $(\mathrm{dg}) k$-algebras, given two $\operatorname{dg}$ categories $\mathcal{A}$ and $\mathcal{B}$, we can form its tensor product $\mathcal{A} \otimes \mathcal{B}$ (and also its derived version $\mathcal{A} \otimes \mathbb{L} \mathcal{B}$ ); see [4, §2.3]. Consider the additive category $\mathrm{Hmo}_{0}$ whose objects are the small dg categories and whose abelian groups of morphisms are given by

$$
\operatorname{Hmo}_{0}(\mathcal{A}, \mathcal{B}):=K_{0} \operatorname{rep}(\mathcal{A}, \mathcal{B}) .
$$

Here, $K_{0}$ is the Grothendieck group [8, Def. 4.5.8] of the full triangulated subcategory $\operatorname{rep}(\mathcal{A}, \mathcal{B})$ of $\mathcal{D}\left(\mathcal{A}^{\text {op }} \otimes^{\mathbb{L}} \mathcal{B}\right)$ whose objects are the bimodules $X$ such that for every object $x$ in $\mathcal{A}$ the $\mathcal{B}$-module $X(-, x)$ is compact in $\mathcal{D}(\mathcal{B})$. Composition in

\footnotetext{
${ }^{2}$ Condition (ii) can be equivalently formulated in terms of a general semi-orthogonal decomposition in the sense of Bondal-Orlov; see [9]. Thm. 6.3(4)].
} 
$\mathrm{Hmo}_{0}$ is induced by the (derived) tensor product of bimodules; see [9, $\left.\S 6\right]$ for details. Note that we have a natural functor

$$
\mathcal{U}_{\mathrm{A}}: \text { dgcat } \longrightarrow \mathrm{Hmo}_{0}
$$

which is the identity on objects and maps a $\operatorname{dg}$ functor $F: \mathcal{A} \rightarrow \mathcal{B}$ to the class in the Grothendieck group $K_{0} \operatorname{rep}(\mathcal{A}, \mathcal{B})$ of the bimodule in $\operatorname{rep}(\mathcal{A}, \mathcal{B})$ naturally associated to $F$. Since the functor $\mathcal{U}_{\mathrm{A}}$ is the identity on objects, we will write, whenever it is clear from the context, $\mathcal{A}$ instead of $\mathcal{U}_{\mathrm{A}}(\mathcal{A})$.

Theorem 2.4. The functor (2.3) is the universal additive invariant; i.e. given any additive category $\mathrm{D}$, we have an induced equivalence of categories

$$
\left(\mathcal{U}_{\mathrm{A}}\right)^{*}: \operatorname{Fun}_{\mathrm{add}}\left(\mathrm{Hmo}_{0}, \mathrm{D}\right) \stackrel{\sim}{\longrightarrow} \operatorname{Fun}_{\mathrm{A}}(\text { dgcat, D }),
$$

where $\mathrm{Fun}_{\mathrm{add}}\left(\mathrm{Hmo}_{0}, \mathrm{D}\right)$ denotes the category of additive functors.

Proof. Let $E$ be an object of $\operatorname{Fun}_{A}($ dgcat, D), i.e. an additive invariant with values in D. Thanks to [9, Thms. 5.3 and 6.3] the functor $E$ factors uniquely through $\mathcal{U}_{\mathrm{A}}$, giving rise to an additive functor $\bar{E}: \mathrm{Hmo}_{0} \rightarrow \mathrm{D}$. If $\eta$ is an element of $\operatorname{Nat}\left(E, E^{\prime}\right)$, i.e. a morphism $\eta: E \Rightarrow E^{\prime}$ of additive invariants, then $\bar{\eta}: \bar{E} \Rightarrow \overline{E^{\prime}}$, with $\bar{\eta}(\mathcal{A}):=\eta(\mathcal{A})$ for every dg category $\mathcal{A}$, is a natural transformation of additive functors. We obtain then a well-defined functor

$$
\overline{(-)}: \operatorname{Fun}_{\mathrm{A}}(\text { dgcat, D }) \longrightarrow \operatorname{Fun}_{\mathrm{add}}\left(\mathrm{Hmo}_{0}, \mathrm{D}\right) \text {. }
$$

Making use of [9, Thms. 5.3 and 6.3], we observe that the functors $\left(\mathcal{U}_{\mathrm{A}}\right)^{*}$ and $\overline{(-)}$ are (quasi-)inverses of each other. This achieves the proof.

Notation 2.6. We will denote by $\underline{k}$ the dg category with a single object and with $k$ as the $(\mathrm{dg}) k$-algebra of endomorphisms.

Lemma 2.7 (9, Lem. 6.5]). Given any $d g$ category $\mathcal{A}$, we have a natural isomorphism of abelian groups

$$
\operatorname{Hom}_{\mathrm{Hmo}_{0}}(\underline{k}, \mathcal{A}) \simeq K_{0}(\mathcal{A})
$$

\section{Canonical isomorphisms}

In this section we provide a description of canonical isomorphisms

$$
\psi^{-}: H C_{0}^{-}(k) \stackrel{\sim}{\longrightarrow} k, \quad \psi_{n}: H C_{2 n}(k) \stackrel{\sim}{\longrightarrow} k, n \geq 0 ;
$$

see Definitions 3.10 and 3.13. Let us start with some arithmetic preliminaries. Consider the integer numbers

$$
y_{l}:=(-1)^{l} \frac{(2 l) !}{l !}, l \geq 0 \quad \text { and } \quad z_{l}:=(-1)^{l-1} \frac{(2 l) !}{2(l !)}, l \geq 1 .
$$

Lemma 3.1. We have the equalities

$$
\begin{array}{lll}
\text { (i) } & (2 l+1) y_{l}=z_{(l+1)}, & l \geq 0, \\
\text { (ii) } \quad-2 z_{l}=y_{l}, & l \geq 1 .
\end{array}
$$

Proof. Equality (ii) is clear. Equality (i) is a simple consequence of the equalities $(2(l+1)) !=(2 l+2)(2 l+1) !$ and $2(l+1) !=(2 l+2) l !$. 
Now, straightforward computations show that in the cyclic bicomplex $C C(k) \underline{6}$, $\S 2.1 .2]$ the maps

$$
b,-b^{\prime}: k^{\otimes(n+1)} \longrightarrow k^{\otimes n}, n \geq 1, \quad(1-t), N: k^{\otimes(n+1)} \longrightarrow k^{\otimes(n+1)}, n \geq 0
$$

are given as follows (where $k^{\otimes m}$ was naturally identified with $k$ ):

$$
\begin{gathered}
b=\left\{\begin{array}{lll}
0 & \text { for } & n \text { odd }, \\
\text { id } & \text { for } & n \text { even, }
\end{array}\right. \\
(1-t)=\left\{\begin{array}{lll}
2 \text { id } & \text { for } & n \text { odd, } \\
0 & \text { for } & n \text { even, }
\end{array}\right.
\end{gathered}
$$

Therefore, in the total complex Tot $C C(k)$, the differential map

$$
d_{m}:(\operatorname{Tot} C C(k))_{m} \longrightarrow(\operatorname{Tot} C C(k))_{(m-1)}, \quad m \geq 1
$$

vanishes for $m$ odd and is surjective for $m$ even. This implies that for $n \geq 0$, we have

$$
H C_{2 n+1}(k)=0 \text { and } H C_{2 n}(k)=Z_{2 n}(\operatorname{Tot} C C(k)) .
$$

Lemma 3.2. For $n \geq 0, Z_{2 n}(\operatorname{Tot} C C(k))$ is the free $k$-module of rank one generated by the canonical cycle

$$
u^{n}:=\left(y_{n} \mathbf{1}, z_{n} \mathbf{1}, \ldots, y_{l} \mathbf{1}, z_{l} \mathbf{1}, \ldots, y_{1} \mathbf{1}, z_{1} \mathbf{1}, y_{0} \mathbf{1}\right) \in(\operatorname{Tot} C C(k))_{2 n} .
$$

Proof. Since by definition $y_{0}=1$, the case $n=0$ is clear. Note that we have

$$
(\operatorname{Tot} C C(k))_{m}=\underbrace{k \oplus k \oplus \cdots \oplus k}_{m+1 \text { factors }}, \quad m \geq 0 .
$$

By fixing the canonical base

$$
\left\{\mathbf{e}_{1}, \mathbf{e}_{2}, \ldots, \mathbf{e}_{r}, \ldots, \mathbf{e}_{m+1}\right\}, \quad \mathbf{e}_{r}:=(0,0, \ldots, \underset{r}{\mathbf{1}}, \ldots, 0)
$$

of the above $k$-modules (3.4), the differential maps

$$
d_{2 n}:(\operatorname{Tot} C C(k))_{2 n} \longrightarrow(\operatorname{Tot} C C(k))_{(2 n-1)}, \quad n \geq 1
$$

can be described by the following $2 n \times(2 n+1)$-matrices:

$$
\left(M_{2 n}\right)_{i, j}:= \begin{cases}(-1)^{(i-1)} & \text { if } i=j \\ 2 & \text { if } j=i+1 \text { and } i \text { is odd } \\ 2 n-i+1 & \text { if } j=i+1 \text { and } i \text { is even } \\ 0 & \text { if } i>j \text { or } j>i+1\end{cases}
$$

Graphically, we have

$$
M_{2 n}=\left(\begin{array}{cccccccc}
1 & 2 & 0 & \cdots & \cdots & \cdots & \cdots & 0 \\
0 & -1 & 2 n-1 & \ddots & & & & \vdots \\
\vdots & \ddots & 1 & 2 & \ddots & & & \vdots \\
\vdots & & \ddots & -1 & \ddots & \ddots & & \vdots \\
\vdots & & & \ddots & \ddots & 3 & \ddots & \vdots \\
\vdots & & & & \ddots & 1 & 2 & 0 \\
0 & \cdots & \cdots & \cdots & \cdots & 0 & -1 & 1
\end{array}\right)_{(2 n) \times(2 n+1)}
$$

In order to prove the lemma for the remaining cases $n \geq 1$, we will now proceed by induction on $n$. 
Case $n=1$ : Using the above notation, the $k$-module $Z_{2}(\operatorname{Tot} C C(k))$ identifies with the kernel of the matrix

$$
M_{2}=\left(\begin{array}{ccc}
1 & 2 & 0 \\
0 & -1 & 1
\end{array}\right)_{2 \times 3} .
$$

A straightforward computation show us that the kernel of $M_{2}$ is given by

$$
\{(-2 a, a, a) \mid a \in k\} \subset k \oplus k \oplus k=(\operatorname{Tot} C C(k))_{2} .
$$

Therefore, since $y_{0}=1, z_{1}=1$ and $y_{1}=-2$, we conclude by taking $a=1$ that the $k$-module (3.5) is the free $k$-module of rank one generated by the cycle $u^{1}$.

Induction step: Suppose that by hypothesis $Z_{2 n}(\operatorname{Tot} C C(k)), n \geq 1$, is the free $k$-module of rank one generated by the cycle $u^{n}$. Using the above notation we then have the equality

$$
Z_{2 n}(\operatorname{Tot} C C(k))=\left\{\left(y_{n} a, z_{n} a, \ldots, y_{1} a, z_{1} a, y_{0} a\right) \mid a \in k\right\} .
$$

We need to describe the kernel of the matrix $M_{2 n+2}$, or equivalently the $k$-module $Z_{2 n+2}($ Tot $C C(k))$. Note that by construction the matrix $M_{2 n}$ identifies with the lower right corner submatrix of $M_{2 n+2}$ of dimension $2 n \times(2 n+1)$. Therefore, the combination of equality (3.6) with a simple computation shows us that the kernel of $M_{2 n+2}$ is given by

$$
\left.\left\{-2(2 n+1) y_{n} a,(2 n+1) y_{n} a, y_{n} a, z_{n} a, \ldots, y_{1} a, z_{1} a, y_{0} a\right) \mid a \in k\right\} .
$$

Thanks to Lemma 3.1 we have the equalities

$$
(2 n+1) y_{n}=z_{n+1} \text { and }-2(2 n+1) y_{n}=y_{n+1},
$$

and so we conclude by taking $a=1$ that the $k$-module (3.7) is the free $k$-module of rank one generated by the cycle $u^{n+1}$. This achieves the proof.

Remark 3.8. The periodicity map $S$ [6, §2.2], which is induced from the truncation of the cyclic bicomplex, sends the generator $u^{n}, n \geq 1$, of $Z_{2 n}(\operatorname{Tot} C C(k))=$ $H C_{2 n}(k)$ to the generator $u^{n-1}$ of $Z_{2 n-2}(\operatorname{Tot} C C(k))=H C_{2 n-2}(k)$. Therefore, thanks to Lemma 3.2. the map $S$ gives rise to isomorphisms

$$
Z_{2 n}(\operatorname{Tot} C C(k)) \stackrel{\sim}{\longrightarrow} Z_{2 n-2}(\operatorname{Tot} C C(k)), \quad u^{n} \mapsto u^{n-1} .
$$

These isomorphisms show us that $u^{n}$ is the unique cycle in (Tot $\left.C C(k)\right)_{2 n}$ which is mapped to $u^{n-1}$ under the periodicity map $S$. Since $u^{0}$ is the unit $\mathbf{1}$ of the base ring $k$, the cycles $u^{n}$ can alternatively be defined by applying the composition of the inverse maps of (3.9) to 1 . This observation justifies the use of the word "canonical" when defining the cycles $u^{n}$ (3.3).

Definition 3.10. The isomorphisms $\psi_{n}, n \geq 0$, are the unique $k$-linear maps that send the canonical generator $u^{n}$ of $H C_{2 n}(k)$ to the unit 1 of the base ring $k$.

Now, recall from [6. Prop. 5.1.9] Milnor's short exact sequence

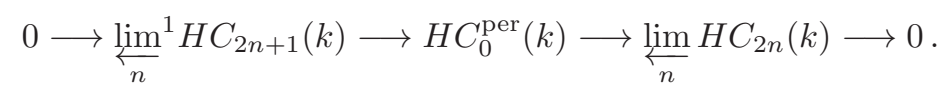

Since $H C_{2 n+1}(k)=0$ for $n \geq 0$, the Mittag-Leffler condition [6, §5.1.10] is automatically fulfilled and so the $\lim ^{1}$-term vanishes. We then obtain a canonical isomorphism

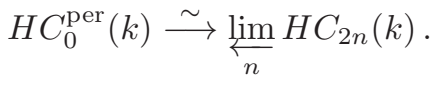


Moreover, the periodicity map $S$ sends the canonical generator $u^{n}$ of $H C_{2 n}(k)$ to the canonical generator $u^{n-1}$ of $H_{2 n-2}(k)$. These facts imply that $H C_{0}^{-}(k)$ is the free $k$-module of rank one generated by the canonical cycle

$$
u^{\infty}:={\underset{\leftarrow}{n}}_{\lim _{n}} u^{n}=\left(\ldots, y_{l} \mathbf{1}, z_{l} \mathbf{1}, \ldots, y_{1} \mathbf{1}, z_{1} \mathbf{1}, y_{0} \mathbf{1}\right) \in\left(\operatorname{ToT} C C^{-}(k)\right)_{0} .
$$

Definition 3.13. The isomorphism $\psi^{-}$is the unique $k$-linear map that sends the canonical generator $u^{\infty}$ to the unit 1 of the base ring $k$.

\section{Absolute characterization}

Proposition 4.1. Let $E: \mathrm{dgcat} \rightarrow \mathrm{Ab}$ be an additive invariant with values in (the additive category of) abelian groups. Then the canonical map

$$
\operatorname{Nat}\left(K_{0}, E\right) \stackrel{\sim}{\longrightarrow} E(\underline{k}), \quad \eta \mapsto \eta(\underline{k})([k])
$$

is an isomorphism of abelian groups. Here, $[k]$ stands for the class of $k$ (as a module over itself) in the Grothendieck group $K_{0}(\underline{k})=K_{0}(k)$ and Nat stands for the abelian group of natural transformations (with group structure given by objectwise addition).

Proof. The functors $K_{0}$ and $E$ are additive invariants and so they belong to the category $\operatorname{Fun}_{A}($ dgcat, $A b)$. Using the functor (2.5) we then obtain an isomorphism

$$
\operatorname{Nat}\left(K_{0}, E\right) \stackrel{\sim}{\longrightarrow} \operatorname{Nat}\left(\overline{K_{0}}, \bar{E}\right), \quad \eta \mapsto \bar{\eta},
$$

which is moreover compatible with the group structure (on both sides) given by objectwise addition. Thanks to Lemma 2.7 the additive functor $\overline{K_{0}}$ is corepresentable in $\mathrm{Hmo}_{0}$ by the object $\underline{k}$. Therefore, since every additive functor is an Ab-functor [1, Def. 6.2.3], the enriched Yoneda Lemma [1, Thm. 8.3.5] furnishes us an isomorphism of abelian groups

$$
\operatorname{Nat}\left(\overline{K_{0}}, \bar{E}\right) \stackrel{\sim}{\longrightarrow} \bar{E}(\underline{k}), \quad \bar{\eta} \mapsto \bar{\eta}(\underline{k})\left(\operatorname{id}_{\underline{k}}\right) .
$$

Note that by construction of $\mathrm{Hmo}_{0}$, the identity id $\mathrm{i}_{k}$ of the object $\underline{k}$ in $\mathrm{Hmo}_{0}$ corresponds to the class $[k]$ of $k$ (as a module over itself) in the Grothendieck group $K_{0}(\underline{k})=K_{0}(k)$. Since $\bar{E}(\underline{k})=E(\underline{k})$ and $\bar{\eta}(\underline{k})=\eta(\underline{k})$, we conclude that the canonical map (4.2) is the composition of the isomorphisms (4.3) and (4.4). This achieves the proof.

Proof of Theorem 1.3. We have the equalities

$$
H C_{0}^{-}(\underline{k})=H C_{0}^{-}(k) \text { and } H C_{2 n}(\underline{k})=H C_{2 n}(k), n \geq 0,
$$

and the functors $H C_{0}^{-}$and $H C_{2 n}, n \geq 0$, are additive invariants with values in abelian groups. Therefore, the canonical isomorphisms (1.4) and (1.5) follow from combining Proposition 4.1 with the isomorphisms $\psi^{-}$and $\psi_{n}$.

Now, let $c h_{n}$ be a Chern character map. Thanks to the agreement property [7, $\S 4.5]$, when we evaluate $c h_{n}$ at $\underline{k}$ we obtain the map

$$
K_{0}(k) \longrightarrow H C_{2 n}(k)
$$

of [6, Thm. 8.3.4] (with $A=k$ ). Note that in order to calculate the value $c h_{n}(\underline{k})([k])$ of the map [4.5) at $[k]$ we can choose for the idempotent $e$ the unit 1 of the base ring $k \simeq \mathcal{M}_{1}(k)$; see [6, §8.3.1]. Therefore, a careful analysis shows that $c h_{n}(\underline{k})([k])$ is precisely the canonical generator $u^{n}$ (3.3) of $H C_{2 n}(k)$; see [6, Lem. 8.3.3]. Using $\psi_{n}$, we conclude that under the canonical isomorphism (1.5), the Chern character 
map $c h_{n}$ is characterized as the natural transformation corresponding to the unit 1 of the base ring $k$.

We now consider the Chern character map $c h^{-}$. Thanks to the agreement property [7, §4.5], when we evaluate $c h^{-}$at $\underline{k}$ we obtain the map

$$
K_{0}(k) \longrightarrow H C_{0}^{-}(k)
$$

of [6. Prop. 8.3.8] (with $A=k$ ). Once again, in order to calculate the value $\operatorname{ch}^{-}(\underline{k})([k])$ of the map (4.6) at $[k]$, we can choose for the idempotent $e$ the unit 1 of the base ring $k \simeq \mathcal{M}_{1}(k)$. In this case we realize that $c h^{-}(k)([k])$ is precisely the canonical generator $u^{\infty}$ (3.12) of $H C_{0}^{-}(k)$. Using $\psi^{-}$, we conclude that under the canonical isomorphism (1.4), the Chern character map $\mathrm{ch}^{-}$is characterized as the natural transformation corresponding to the unit $\mathbf{1}$ of the base ring $k$.

\section{Relative CHARACTERIZATION}

Proof of Theorem 1.7, By construction, see [7, Defs. 4.4.1-4.4.2] and diagram [6. (11.4.3.3)] (with $n=0$ ), we have $S^{(n-m)} \circ c h_{n}=c h_{m}$. Now, recall that the periodicity map

$$
S: H C_{2 n} \Rightarrow H C_{2 n-2},
$$

when evaluated at $\underline{k}$, sends the canonical generator $u^{n}\left(\underline{3.3)}\right.$ of $H C_{2 n}(k)=H C_{2 n}(\underline{k})$ to the canonical generator $u^{n-1}$ of $H_{2 n-2}(k)$; see [6, Rk. 2.2.2]. We then have the following commutative diagram:

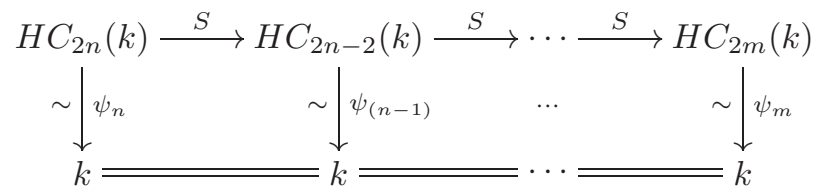

Thanks to Theorem 1.3 the Chern character map $c h_{n}$ corresponds, under the canonical isomorphism (1.5), to the unit 1 of the base ring $k$. Therefore, using the above commutative diagram (5.1), we conclude that $c h_{n}$ is in fact the unique natural transformation such that $S^{(n-m)} \circ c h_{n}=c h_{m}$. This shows item (i).

Let us now show item (ii). Once again by construction, the composition (1.8) equals the Chern character map $c h_{m}$; see [6, Prop. 8.3.8]. Recall from [6, §5.1] that the natural transformation

$$
I: H C_{0}^{-} \Rightarrow H C_{0}^{\text {per }}
$$

is the identity. We then have the following commutative diagram:

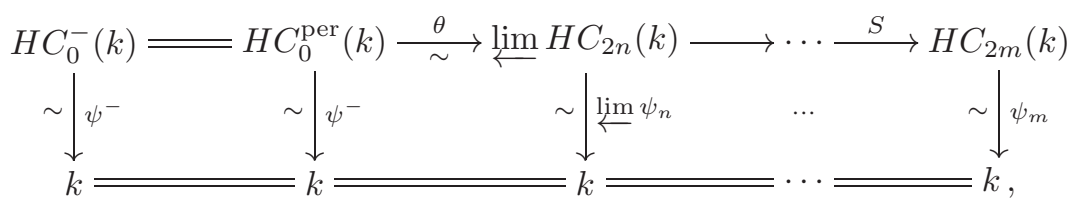

where $\theta$ is the isomorphism (3.11). Thanks to Theorem 1.3 the Chern character map $c h^{-}$corresponds, under the canonical isomorphism (1.4), to the unit 1 of the base ring $k$. Therefore, using Lemma 5.3 Proposition 4.1, and the above commutative diagram (5.2), we conclude that $c h^{-}$is in fact the unique natural transformation such that the composition (1.8) equals the Chern character map $c h_{m}$. This shows item (ii). 
Lemma 5.3. The functor

$$
\underset{n}{\lim } H C_{2 n}: \text { dgcat } \longrightarrow \mathrm{Ab}
$$

is an additive invariant.

Proof. Condition (i) is clear. Condition (ii) follows from the fact that the functors $H C_{2 n}, n \geq 0$, are additive invariants and that finite sums agree with finite products in $\mathrm{Ab}$.

\section{ACKNOWLEDGMENTS}

The author is very grateful to Paul Balmer, Andrew Blumberg, David Gepner and Christian Haesemeyer for stimulating conversations and to the anonymous referee for comments which greatly improved the paper. He would also like to thank the Department of Mathematics at UCLA, where part of this work was carried out, for its hospitality and excellent working conditions.

\section{REFERENCES}

[1] F. Borceux, Handbook of categorical algebra. 2. Categories and Structures. Encyclopedia of Mathematics and its Applications, vol. 51. Cambridge Univ. Press, 1994. MR 1313497 (96g:18001b)

[2] S. S. Chern, Characteristic classes of Hermitian manifolds. Ann. of Math. (2) 47(1) (1946), 85-121. MR0015793 (7:470b)

[3] A. Connes, Noncommutative differential geometry. Publ. Math. de l'IHÉS 62 (1985), 257360. MR0823176 (87i:58162)

[4] B. Keller, On differential graded categories. International Congress of Mathematicians (Madrid), Vol. II (2006), 151-190. Eur. Math. Soc., Zürich. MR.2275593 (2008g:18015)

[5] - On the cyclic homology of exact categories. J. Pure Appl. Algebra 136(1) (1999), 1-56. MR 1667558 (99m:18012)

[6] J.-L. Loday, Cyclic homology. Grundlehren der Mathematischen Wissenschaften 301 (1992). Springer-Verlag, Berlin. MR1217970 (94a:19004)

[7] R. McCarthy, The cyclic homology of an exact category. J. Pure Appl. Algebra 93(3) (1994), 251-296. MR1275967 (95b:19002)

[8] A. Neeman, Triangulated categories. Ann. Math. Studies, vol. 148. Princeton Univ. Press, 2001. MR 1812507 (2001k:18010)

[9] G. Tabuada, Invariants additifs de dg-catégories. Int. Math. Res. Not. 53 (2005), 3309-3339. MR2196100 (2006k:18018)

[10] Corrections à "Invariants additifs de dg-catégories", Int. Math. Res. Not., article ID rnm 149 (2007). MR2377018(2008j:18011)

Departamento de Matemática e CMa, Faculdade de Ciências e Tecnologia, Universidade Nova de Lisboa, Quinta da Torre, 2829-516 Caparica, Portugal

E-mail address: tabuada@fct.unl.pt 